\title{
Optimum Power in a Multi-Span DWDM System Limited by Non-Linear Effects
}

\author{
Jose Alfredo Alvarez-Chavez'1,2*, Rafael Sanchez-Lara3, Jaime Rafael Ek-Ek², \\ Herman Leonard Offerhaus ${ }^{1}$, Manuel May-Alarcon'3, Victor Golikov ${ }^{3}$ \\ ${ }^{1}$ Optical Sciences Group, University of Twente, Enschede, The Netherlands \\ ${ }^{2}$ Instituto Politecnico Nacional-Centro de Investigacion e Innovacion Tecnologica, Catarina, Mexico \\ ${ }^{3}$ Facultad de Ingenieria, Universidad Autonoma del Carmen, Campeche, Mexico \\ Email: ${ }^{\star j}$.a.alvarezchavez@utwente.nl
}

How to cite this paper: Alvarez-Chavez, J.A., Sanchez-Lara, R., Ek-Ek, J.R., Offerhaus, H.L., May-Alarcon, M. and Golikov, V. (2018) Optimum Power in a Multi-Span DWDM System Limited by Non-Linear Effects. Optics and Photonics Journal, 8, 337-347.

https://doi.org/10.4236/opj.2018.812029

Received: November 6, 2018

Accepted: December 9, 2018

Published: December 12, 2018

Copyright $\odot 2018$ by authors and Scientific Research Publishing Inc. This work is licensed under the Creative Commons Attribution International License (CC BY 4.0). http://creativecommons.org/licenses/by/4.0/

\begin{abstract}
Limitations imposed by Four-Wave Mixing (FWM), Amplified Spontaneous Emission (ASE), dispersion and Stimulated Raman Scattering (SRS) on a multi-spam DWDM system are theoretically studied. In this work, expression for the linear dispersion parameter $\mathrm{D}$ has been defined as a function of number of channels in order to separate FWM and SRS effects and calculates both maximum fibre length and optimum power. Additionally, our simulation results consider the effect of ASE noise from Erbium Doped Fibre Amplifiers (EDFAs). This theoretical analysis yields a set of design criteria from optimized multi-span DWDM systems.
\end{abstract}

\author{
Keywords \\ DWDM, FWM, ASE, SRS, Dispersion, EDFA
}

\section{Introduction}

Long distance high capacity optical transmission is achieved using optical fibre links in backbone of terrestrial and transoceanic communication systems. Linear and non-linear effects limit the capacity. Among the main non-linear effects are Four-Wave Mixing (FWM), Stimulated Raman Scattering (SRS), Stimulated Brillouin Scattering (SBS), Self-Phase Modulation (SPM), and Cross-Phase Modulation (XPM). Furthermore, Amplified Spontaneous Emission (ASE) noise from Erbium-Doped Fibres Amplifiers (EDFAs) is present. Dense Wavelength Division Multiplexing (DWDM) technology is used to make maximum use of the total bandwidth of single mode optical fibres [1] [2]. The effect of dispersion and nonlinearities limit the performance for transmission over long distances at data rates greater than 2.5 Gbits/s. EDFAs are used to compensate fibre losses 
and to increase the transmission distance, causing at the same time, an increase of ASE noise and nonlinearities. Different kinds of fibres have been designed to reduce the effect of dispersion and nonlinearities [3] [4] [5]. There are numerous studies on nonlinear impairments showing the importance and complexity of such phenomena for DWDM [6]-[11].

In this paper, design parameters for multi-span DWDM system limited by FWM, SRS and ASE noise are proposed. The dispersion parameter limit is also calculated, and the optimum power transmission per channel is evaluated for different number of channels or inter-channel spacing. Additionally, our analysis shows different spectral regions where each nonlinearity is dominant, and how to find intersections where general spectral limits can be obtained, taking into account the combined effects of FWM, SRS, and ASE noise.

\section{Model}

The multi-spam DWDM model used is show in Figure 1.

Where $N$ is the number of channels, $L_{a}$ is the optical amplifier spacing, $L$ is the system length, $M$ is the number of segments, $A_{1}$ and $A_{m-1}$ are the first and last EDFA respectively, $f_{1}$ and $f_{N}$ are the first and last frequency channel.

A total spectral bandwidth $W=f_{N}-f_{1}=3.75 \mathrm{THz}(30 \mathrm{~nm})$ within C-band is considered. The WDM density parameter $\Delta f$ is a function of the number of channels $N$ and can be expressed as:

$$
\Delta f=f_{i}-f_{i-1}=W /(N-1)
$$

The central frequency $f$ is given by:

$$
f=\frac{f_{1}+f_{N}}{2}=193 \mathrm{THz}[1550 \mathrm{~nm}]
$$

$\mathrm{C}$ bands central frequency is a well-known value. At $1550 \mathrm{~nm}$ its equivalent to $193 \mathrm{THz}$ approximately ( $f=c /$ wavelength).

The FWM signal is located at a frequency $f_{n}=f_{i}+f_{j}-f_{k}(i, j \neq k)$ and it is given by the mixing of three signal frequencies $f_{i}, f_{j}$ and $f_{k}$ co-propagating through a single-mode fibre, which generates a new wave frequency (FWM signal) by means of nonlinear interaction. The power of the FWM signal at the $f_{n}$ frequency is given by [10] [12]:

$$
P_{i j k}=k^{2} * P_{i} * P_{j} * P_{k} * \eta_{i j k} * d_{i j k}^{2}\left(\frac{M * L_{e f f}}{A_{e f f}}\right)^{2} \mathrm{e}^{-a * L_{a}}
$$

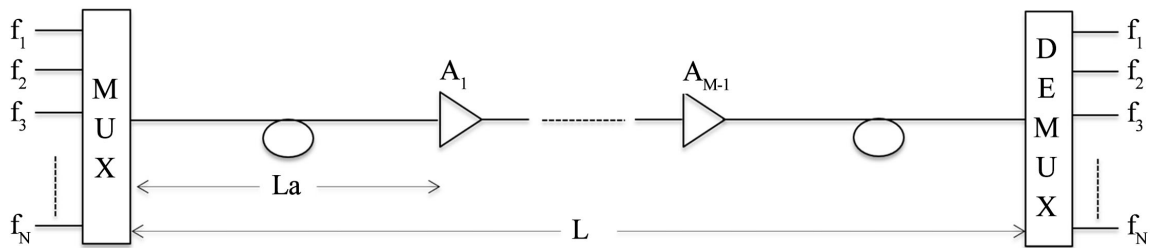

Figure 1. DWDM architecture. 


$$
k=\frac{32 * \pi^{3} * \chi^{(3)}}{\eta^{2} * c * \lambda}
$$

where, $\lambda$ is wavelength, $c$ is the light speed in vacuum, $\eta$ is the core refractive index, $a$ is the linear loss coefficient, $P_{i, j, k}$ are launched input power per channel, $L_{e f f}=\left(1-\exp \left(-a * L_{a}\right)\right) / a$ is the fibre effective length, $A_{\text {eff }}$ is the effective area of the guided mode, $d$ is the degeneracy factor $(d=3$ for $i=j$, $d=6$ for $i \neq j), \quad \chi^{(3)}$ is the third-order nonlinear electric susceptibility $(6 \times$ $10^{-14} \mathrm{~m}^{3} \mathrm{~W}^{-1}$ ) and $\eta_{i j k}$ is the FWM efficiency, given by:

$$
\eta_{i j k}=\left(\frac{a^{2}}{a^{2}+\Delta \beta_{i j k}^{2}}\right)\left(1+\left(\frac{4 \mathrm{e}^{-a * L_{a}}}{\left(1-\mathrm{e}^{-a * L_{a}}\right)^{2}}\right) \sin ^{2}\left(\frac{\Delta \beta_{i j k} * L_{a}}{2}\right)\right)
$$

where $\Delta \beta_{i j k}$ represents the phase mismatch which may be expressed in terms of signal frequency differences and fibre dispersion as:

$$
\Delta \beta_{i j k}=\left(\frac{2 * \pi * \lambda^{2}}{c}\right)\left(\left|f_{i}-f_{k}\right|\left|f_{j}-f_{k}\right|\right)\left(D+\frac{\mathrm{d} D}{\mathrm{~d} \lambda}\left(\frac{\lambda^{2}}{2 * c}\right)\left(\left(f_{i}-f_{k}\right)+\left(f_{j}-f_{k}\right)\right)\right)
$$

here, $D$ is the fibre chromatic dispersion coefficient which is wavelength dependent. In a multi-wavelength system with $N$ wavelength channels of equal channel spacing, the total FWM power generated at the frequency $f_{n}$ may be expressed as a summation:

$$
P_{n}=\sum_{f_{n}=f_{i}+f_{j}-f_{k}} P_{i j k}=k^{2} * P_{s} * \mathrm{e}^{-a * L_{a}}\left(\frac{M * L_{e f f}}{A_{e f f}}\right)^{2} \sum_{f_{n}=f_{i}+f_{j}-f_{k}} \eta_{i j k} * d_{i j k}^{2}
$$

where the summation is made over all the relevant combinations which satisfy the relation: $f_{n}=f_{i}+f_{j}-f_{k}(i, j j \neq k)$.

We can define the term $Y$ indicating the maximum values of the summation $\eta_{i j k} d_{i j k}^{2}$ for overall channels as follow:

$$
Y=\operatorname{MAX}\left(\sum \eta_{i j k} * d_{i j k}^{2}\right), i, j, k=1,2, \cdots, N
$$

In a system with equal power per channel $\left(P_{i}=P_{i}=P_{k}=P_{s}\right)$, the FWM power on the worst affected channel $(n=w)$ is given by:

$$
P_{w}=k^{2} * P_{s}\left(\frac{M * L_{e f f}}{A_{e f f}}\right)^{2} Y * \mathrm{e}^{-a \cdot L_{a}}
$$

For the case of a system with different power levels, Equation (9) would only affect the $Y$ term by adding each different power per channel. In such case $Y$ would be:

$$
Y=\operatorname{MAX}\left(\sum P_{i j k} * \eta_{i j k} * d_{i j k}^{2}\right)
$$

Nevertheless, for practical purposes all channels are considered to have equal power.

FWM can be considered as an interference signal, and supposing that the required optical signal to interference ratio is $20 \mathrm{~dB}$ (typical value) at the receiver 
to ensure no appreciable performance penalty [1], the maximum launched power per channel $P_{m}$ can be obtained considering the worst affected channel and it can be approximated as follows:

$$
\begin{gathered}
\frac{P_{m} * \mathrm{e}^{-a * L_{a}}}{P_{w}}=100 \\
P_{m}=\frac{A_{e f f}}{M * L_{e f f}} \sqrt{\frac{1}{100 * k^{2} * Y}}
\end{gathered}
$$

The effect of ASE noise due to inline amplifiers is estimated next. The total amount of ASE noise power at the receiver increases with the number of amplifiers. Therefore, in long distance amplified systems the ASE noise dominates over other noise sources (shot noise and thermal noise), and the Bit Error Rate $(B E R)$ performance is mainly determined by optical signal-to-noise ratio $(O S N R)$. The SNR can be estimated from a simple Gaussian approximation in [13]:

$$
S N R=\frac{Q^{2}+Q \sqrt{\frac{B_{o}}{B_{e}}}}{\sqrt{\frac{B_{o}}{B_{e}}}}
$$

where $B_{o}$ is the bandwidth of the optical filter, $B_{e}$ is the electrical bandwidth of the receiver and $Q$ a parameter related to $B E R$ given by:

$$
B E R=\frac{1}{2} \operatorname{erfc}\left(\frac{Q}{\sqrt{2}}\right) \approx \frac{e^{-\frac{Q^{2}}{2}}}{Q \sqrt{2 * \pi}}
$$

The filter bandwidth should be large enough to pass the entire frequency contents of the selected channel but, at the same time, small enough to block the neighbouring channels and noise, typically $B_{o} \sim B$ and it represents the minimum optical bandwidth to avoid blocking signal [1] and $B_{e}<B$, in our simulation we take $B_{o} \sim 2 B$ and $B_{e} \sim B / 2$ to provide sufficient margin. To achieve a $B E R$ of $1 \times 10^{-12}, Q=7$ and $S N R=15.75(11.97 \mathrm{~dB})$ from Equations (8) (9). Assuming an $S N R=20 \mathrm{~dB}$ (typical value), the total ASE noise at the detector can be expressed as [1] [14]:

$$
P_{s p}=2 * n_{s p} *(G-1) * h * f * B_{o} * M
$$

where $h$ is Plancks constant $\left(6.63 \times 10^{-34} \mathrm{~J} \cdot \mathrm{s}\right), f$ is the centre frequency, $G$ is the gain of the amplifier, $n_{s p}$ is the population inversion parameter and $2 n_{s p}$ is the amplifier noise factor and its minimum value is 2 for an ideal amplifier [1]. Considering equal amplifier spacing $\left(M=L / L_{a}\right)$ and that amplifier gain $G$ compensates for fibre loss as:

$$
G=E * \mathrm{e}^{a * L_{a}}
$$

where $E$ is a factor accounting for possible losses introduced by fibre splices and other optical components inserted in the transmission line, we can assume the 
value of 1 for practical purposes. The minimum power per channel to ensure the required $\mathrm{SNR}(20 \mathrm{~dB})$ can then be obtained as:

$$
P=200 * n_{s p} *(G-1) * h * f * B_{o} * L / L_{a}
$$

The FWM effect imposes an upper limit and ASE imposes, on the contrary, a minimum limit to the power per channel value when the transmission distance is increased. Therefore, the intersection between $P$ and $P_{m}$ provides the maximum value of transmission distance $\left(L=L_{\max }\right)$ and $P_{o-f w m}=P=P_{m}$ is the optimum transmission power as shown below:

$$
\begin{gathered}
L_{\max f w m}=\left\{\frac{A_{e f f} * L_{a}^{2}}{200 * h * f * n_{s p} * B_{o} *\left(E * \mathrm{e}^{a * L_{a}}-1\right) * L_{e f f}} *\left[\sqrt{\frac{1}{100 * k^{2} * Y}}\right]\right\}^{1 / 2} \\
P_{o f f w m}=\left\{200 * h * f * n_{s p} * B_{o} *\left(E \mathrm{e}^{a L_{a}}-1\right)\left(1 / L_{e f f}\right)\left[\sqrt{\frac{1}{100 * k^{2} * Y}}\right]\right\}^{1 / 2}
\end{gathered}
$$

Equations (13) and (14) are valid only for a systems limited by FWM and ASE noise.

Next it is important to calculate the dispersion limit. The limit of transmission distance for Intensity Modulation with Direct Detection (IM/DD) systems due to dispersion effect is given by the following expression [14]:

$$
L_{D}=\frac{c}{2 * B^{2} * \lambda^{2} *|D|}
$$

The effect of SRS-ASE can be considered in a similar way. In this case, an upper and lower limit can be obtained for the power per channel due to SRS-ASE. To do this, a worst-case neglecting walk-off effect and dispersion are considered. The maximum power to ensure a $S N R$ degradation of less than $0.5 \mathrm{~dB}$ in the worst channel is given by [15]:

$$
P_{\text {max } \_r s s}=\frac{8.7 * 10^{12}}{N(N-1) M * \Delta f * L_{e f f}}
$$

The intersection between $P_{\text {max } \_s r s}$ and Equation (12) gives a maximum value of transmission distance considering SRS and ASE noise:

$$
L_{\text {max }_{-} s r s}=\sqrt{\frac{8.7 * 10^{12} * L_{A}^{2}}{200 * h * f * n_{s p} * B_{o} * N(N-1)\left(E * \mathrm{e}^{\alpha * L_{a}}-1\right) \Delta f * L_{e f f}}}
$$

So, the optimum transmission power for a system limited only by SRS and ASE noise is given by:

$$
P_{o_{-} s r s}=41.7 * 10^{6} * \sqrt{\frac{h * f * n_{s p} * B_{o}\left(E * \mathrm{e}^{a * L_{a}}-1\right)}{N * W * L_{e f f}}}
$$

The intersection between $P_{\text {max_srs }}$ and Equation (21) gives a maximum value of transmission distance considering SRS and ASE noise:

Equations (13) and (17) can be used to evaluate the optimal length for multi-span DWDM systems limited by FWM and SRS separately. Then, we can ana- 
lyse overlapping regions where both nonlinearities fix the same optimal length. This intersection can provide us with a more general limit for the maximum length in the DWDM system considering the combined effects of FWM, SRS, and ASE respectively. With this procedure one can obtain the spectral limits of a DWDM system without considering the simultaneous analysis of both nonlinearities [16]. In this case, we have to be clear take that we are not interested to study the gradual degradation process through the fibre due to FWM an SRS, but rather the final degradation due to both nonlinearities in the worst channel.

\section{Simulation Results and Discussion}

The amplifier spacing $\left(L_{a}\right)$ can be calculated according to typical values of receiver sensitivity as: $S=-24 \mathrm{dBm}, \alpha=0.25 \mathrm{~dB} / \mathrm{km}$ (a in dB), $P_{T}=1 \mathrm{~mW}$.

A value of $L_{a}=75 \mathrm{~km}\left(L_{a}<\left(P_{T}-S\right) / \alpha\right)$ and a bit rate $B=2.5$ Gbps per channel is considered in all our simulations.

Figure 2 shows the FWM power per channel (Equation (4)) evaluated for different values of $D$, with 31 channels and $1 \mathrm{~nm}$ channel spacing. As we can see, the FWM power depends on the location of the zero dispersion wavelength. At $D=0 \mathrm{ps} /((\mathrm{km} \cdot \mathrm{nm}))$ the maximum FWM power in the central channel is obtained due to the maximum efficiency $\eta_{i j k}$ is obtained too, it agrees with [12]. At $D \neq 0$ the FWM power decreases. If the zero dispersion wavelength is far of the WDM signal bandwidth, the right or left extreme channels suffer most (depending on the sign of $\mathrm{D}$ ).

Figure 3 and Figure 4 show the maximum length considering the combined effects of SRS-ASE, FWM-ASE and Dispersion in a separated form for a 30- and 70-channel system, respectively. Three intervals or domains can be identified by the intersection of the curves, SRS-ASE, FWM-ASE and Dispersion domain.

The evolution of these intersection can be approximated through the following expressions:

FWM-ASE interval domination.

$$
D<8.9 * 10^{-5} * N^{2}+0.011 * N+1.1
$$

Figure 5 shows the evolution of the optimal power for different intervals in agreement with Equation (19). As it can be seen, they are higher than the power threshold for SRS and SBS (Stimulated Brillouin Scattering) [17] [18] [19] [20] [21].

Table 1 is obtained via Equations (13) (14) and (19). It shows the maximum value of optimum power $\left(P_{o_{f} f w m}\right)$ and maximum length $\left(L_{\max }\right)$ in a FWM-ASE domain. $P_{o f f w m}$ and $L_{\max }$ decrease depending of the interval of $D$, and value of $D$.

For a DWDM system with $N=100$ channels $(\Delta f=37.8 \mathrm{GHz})$, the maximum value of $P_{o_{f} f w m}$ is $0.15 \mathrm{~mW}$ with a value of $D=3.09 \mathrm{ps} / \mathrm{km} \cdot \mathrm{nm}$, and it can decrease depending on $\mathrm{D}$.

$P_{o \text { fwrm }}$ varies in a range of $0.78-0.15 \mathrm{~mW}$ for a range $N$ of $1.21-3.09$ $\mathrm{ps} / \mathrm{km} \cdot \mathrm{nm}$ up to $N=100$ channels.

$S R S$-ASE interval domination. 


$$
8.9 * 10^{-5} * N^{2}+0.011 * N+1.1<D<-0.00066 * N^{2}+0.18 * N+3.8
$$

Figure 6 shows a curve of the evolution of the optimal power for different number of channels in agreement with (20).

Table 2 is obtained using Equations (17) (18) and (20). It shows the maximum value of optimum power $\left(P_{o_{s} s r s}\right)$ and maximum length $\left(L_{\max }\right)$ in a SRS-ASE domain. $P_{o_{-} s r s}$ and $L_{\max }$ decrease depending on the value of the design parameters $(N, D, \Delta f)$.

For a DWDM system of $N=100$ channels $(\Delta f=37.8 \mathrm{GHz})$, the maximum value of $P_{o_{-} s s}$ is $0.005 \mathrm{~mW}$ with a value between $D=3.09$ to $15.2 \mathrm{ps} / \mathrm{km} \cdot \mathrm{nm}$, and it is constant in all interval of $D$. The interval of $D$ is higher than FWM-ASE case, but the level of optimum power is lower.

Finally, the interval dominated by the effect of linear dispersion $\mathrm{D}$ is given by:

$$
D>-0.00066 * N^{2}+0.18 * N+3.8
$$

Table 1. $P_{o_{-} f w m}(\mathrm{~mW})$ and $L_{\max }(\mathrm{km})$ in FWM-ASE domain, for different design parameters $N$ (channels), $\Delta f(\mathrm{GHz}), D(\mathrm{ps} / \mathrm{km} \cdot \mathrm{nm})$.

\begin{tabular}{ccccc}
\hline$N$ & $D<$ & $\Delta f$ & $L_{\max }<$ & $P_{o_{-} f w m}<$ \\
\hline 10 & 1.21 & 416 & 2044 & 0.78 \\
20 & 1.35 & 197 & 1407 & 0.33 \\
30 & 1.51 & 129 & 1157 & 0.31 \\
40 & 1.68 & 96 & 1002 &. \\
50 & 1.87 & 76 & 898 &. \\
60 & 2.08 & 63.5 & 818 & 0.21 \\
70 & 2.30 & 54.3 & 757 &. \\
80 & 2.54 & 47.4 &. & 0.18 \\
90 & 2.81 & 42.1 &. &. \\
100 & 3.09 & 37.8 & 634 & 0.15 \\
\hline
\end{tabular}

Table 2. $P_{o_{-} s r s}(\mathrm{~mW})$ and $L_{\max }(\mathrm{km})$ in SRS-ASE domain, for different design parameters $N$ (channels), $\Delta f(\mathrm{GHz}), D(\mathrm{ps} / \mathrm{km} \cdot \mathrm{nm})$.

\begin{tabular}{ccccc}
\hline $\boldsymbol{N}$ & $\boldsymbol{D}$ & $\boldsymbol{\Delta}$ & $\boldsymbol{L}_{\max }$ & $\boldsymbol{P}_{\text {o_srs }}$ \\
\hline 10 & $1.21 \rightarrow 5.53$ & 416 & 2044 & 0.0160 \\
20 & $1.35 \rightarrow 7.13$ & 197 & 1407 & 0.0113 \\
30 & $1.51 \rightarrow 8.60$ & 129 & 1157 & 0.0092 \\
40 & $1.68 \rightarrow 9.94$ & 96 & 1002 & 0.0080 \\
50 & $1.87 \rightarrow 11.15$ & 76 & 898 & 0.0071 \\
60 & $2.08 \rightarrow 12.22$ & 63.5 & 818 & 0.0065 \\
70 & $2.30 \rightarrow 13.16$ & 54.3 & 757 & 00060 \\
80 & $2.54 \rightarrow 13.97$ & 47.4 &. & 0.0056 \\
90 & $2.81 \rightarrow 14.65$ & 42.1 &. & 0.0053 \\
100 & $3.09 \rightarrow 15.20$ & 37.8 & 634 & 0.0050 \\
\hline
\end{tabular}




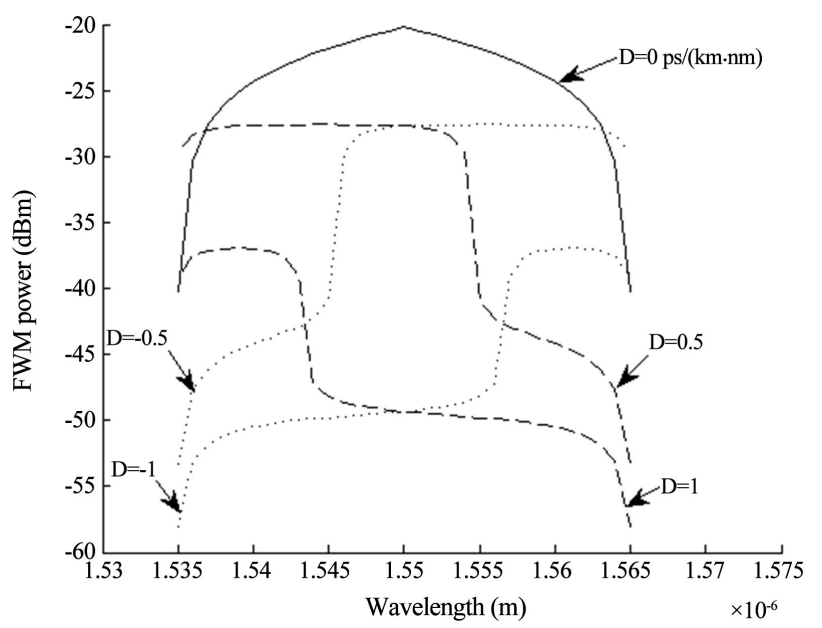

Figure 2. FWM power per channel for positive and negative values of D.

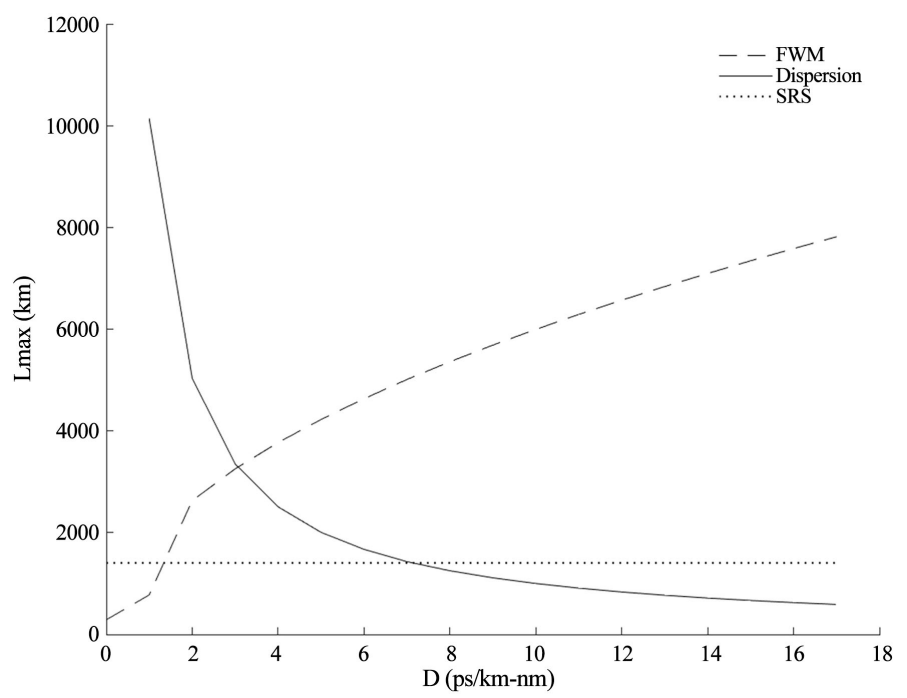

Figure 3. Limitation due to FWM-SRS-ASE-Dispersion for $N=20$ channels.

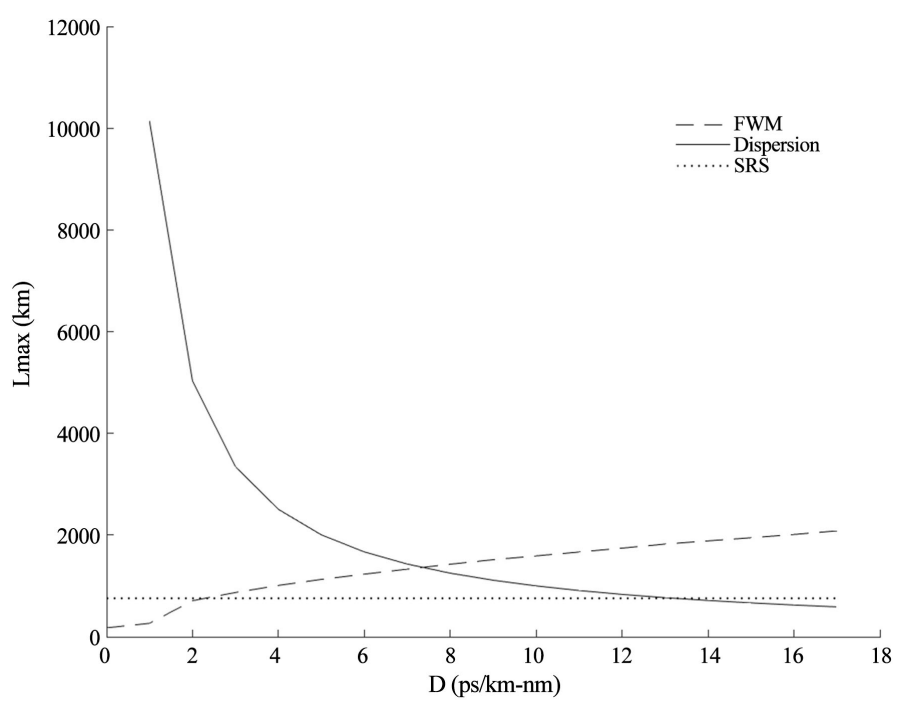

Figure 4. Limitation due to FWM-SRS-ASE-Dispersion for $N=70$ channels. 


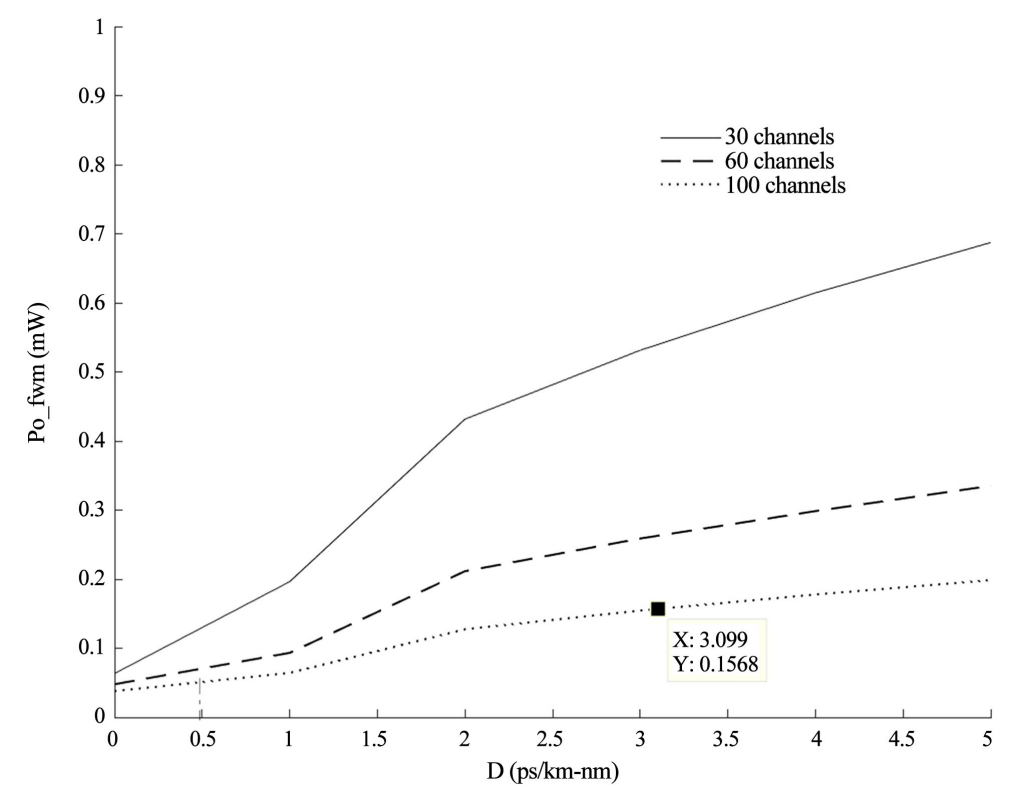

Figure 5. Optimum power as a function of D, for a system limited by FWM y ASE, for 30, 60 and 100 channels.

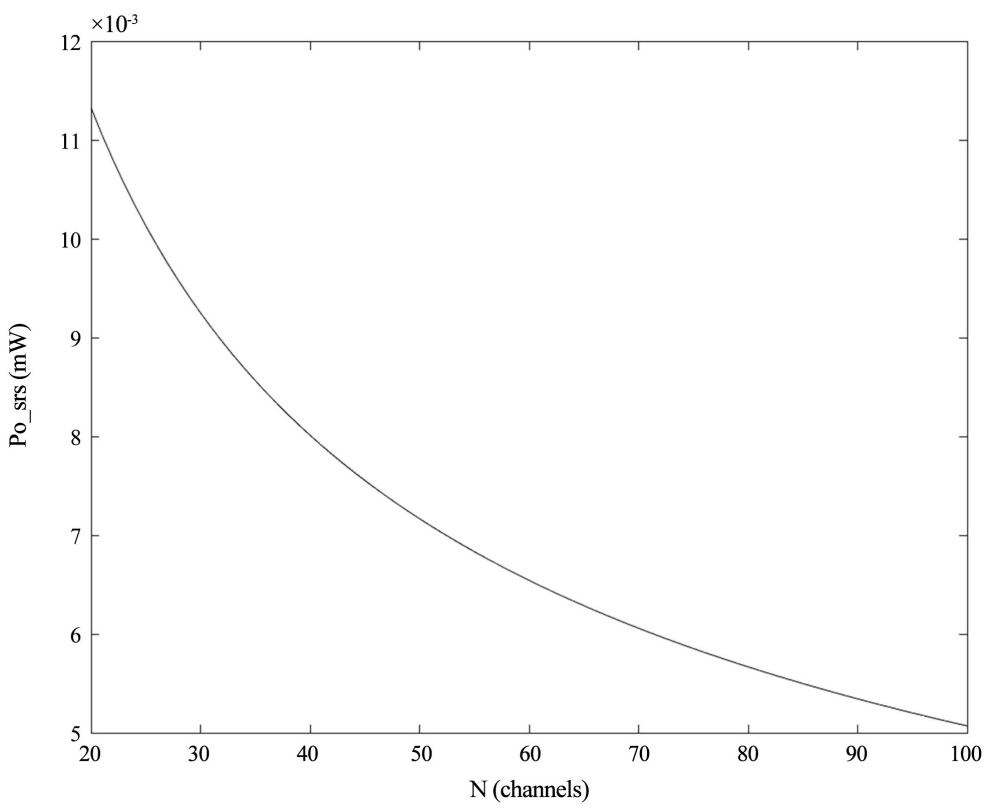

Figure 6. Optimum power as a function of $N$, for a system limited by SRS and ASE.

\section{Conclusion}

We have explored the limitations of a multi-span DWDM system imposed by FWM, ASE, dispersion and SRS for up to 100 channels. We have determined the conditions under which DWDM systems will be limited mainly by FWM and ASE, or SRS and ASE separately and altogether. In particular, for the FWM-ASE nonlinearities, the optimum power transmission per channel was calculated. The effect of SBS can be neglected because the power needed for these nonlinear effects to appear above their threshold is not reached and Brillouin gain band- 
width is too short. The optimum link length that can be achieved for these systems was calculated from the channel number or inter-channel spacing and the total dispersion based on the crossover of the predicted FWM, SRS and dispersion limit. In particular, for channel spacing higher than $125 \mathrm{GHz}$, the SRS-ASE nonlinearity becomes dominant in comparison to the FWM-ASE effect, but at the same time, according to the proposed methodology, one can adjust the design parameters in order to obtain a same effect of the FWM and SRS nonlinearities including the ASE noise respectively. Our results are of great interest for the design of novel optimized multi-span DWDM systems.

\section{Acknowledgements}

The authors are grateful towards UNACAR, IPN-CIITEC and CONACYT from Mexico, and Optical Sciences Group at Twente University in the Netherlands.

\section{Conflicts of Interest}

The authors declare no conflicts of interest regarding the publication of this paper.

\section{References}

[1] Agrawal, G.P. (2010) Fiber-Optic Communication Systems. John Wiley \& Sons. https://doi.org/10.1002/9780470918524

[2] Rostami, A., Rahbari, J. and Andalib, A. (2013) Investigation of Power Penalty in WDM Systems for Dispersion Managed Fibers. Optik, 124, 2072-2075. https://doi.org/10.1016/j.ijleo.2012.06.065

[3] Karfaa, Y.M., et al. (2007) Effects of Four-Wave Mixing Crosstalk in WDM Networks on the Transmitted Optical Frequencies and Wavelengths of Channels for Various Fiber Types. Asia-Pacific Conference on Applied Electromagnetics APACE, Melaka, 1-5. https://doi.org/10.1109/APACE.2007.4603976

[4] Djordjevic, I.B. (2001) Transmission Limitation of WDM Transmission Systems with Dispersion Compensated Links in the Presence of Fiber Nonlinearities. Conference on Telecommunications in Modern Satellite, Cable and Broadcasting Service TELSIKS, 5 th International, Nis, 19-21.

[5] Rana, Md.M. and Khan, M.A.G. (2012) Dense Wavelength Division Multiplexing Optical Network System. International Journal of Electrical and Computer Engineering, 2, 203-206

[6] Kowalewski, M., et al. (2000) Nonlinear Interactions in Wavelength-Multiplexed Optical Fibre Telecommunications Systems. Journal of Optics A, 2, 319-326. https://doi.org/10.1088/1464-4258/2/4/314

[7] Gurmeet Kaur, M.I. Singh, M. and Patterh, S. (2009) Theoretical Investigations of the Combined Effect of Fibre Nonlinearities, Amplifier and Receiver Noise in a DWDM Transmission System. Journal of Optical and Fiber Communications Research, 6, 1-10. https://doi.org/10.1007/s10297-009-9002-8

[8] Wu, M. and Way, W.I. (2004) Fiber Nonlinearity Limitations in Ultra-Dense WDM Systems. IEEE Journal of Lightwave Technology, 22, 1483-1497. https://doi.org/10.1109/JLT.2004.829222

[9] Louchet, H., et al. (2005) Analytical Model for the Design of Multispan DWDM 
Transmission Systems. IEEE Photonics Technology Letters, 17, 247-249. https://doi.org/10.1109/LPT.2004.838142

[10] Maeda, M.W., et al. (1990) The Effect of Four-Wave Mixing in Fibers on Optical Frequency-Division Multiplexed Systems. IEEE Journal of Lightwave Technology, 8, 1402-1408. https://doi.org/10.1109/50.59171

[11] Ellis, D., Mccarthy, M.E., Alkhateeb, M.A.Z., Sorokina, M. and Doran, N.J. (2017) Performance Limits in Optical Communications Due to Fiber Nonlinearity. Advances in Optics and Photonics, 9, 429-503. https://doi.org/10.1364/AOP.9.000429

[12] Inoue, K. (1992) Four Wave Mixing in an Optical Fiber in the Zero-Dispersion Wavelength Region. IEEE Journal of Lightwave Technology, 10, 1533-1561. https://doi.org/10.1109/50.184893

[13] Marcuse, D. (1990) Derivation of Analytical Expressions for the Bit-Error Probability in Lightwave Systems with Optical Amplifiers. IEEE Journal of Lightwave Technology, 8, 1816-1823.

[14] Henry, P.S. (1985) Lightwave Primer. IEEE Journal of Quantum Electronics, 21, 1862-1879. https://doi.org/10.1109/JQE.1985.1072601

[15] Chraplyvy, A.R. and Tkach, R.W. (1993) What Is the Actual Capacity of Single-Mode Fibers in Amplified Lightwave Systems. IEEE Photonics Technology Letters, 5, 666-668. https://doi.org/10.1109/68.219704

[16] Vanholsbeeck, F., Coen, S., et al. (2005) Coupled-Mode Analysis of Stimulated Raman Scattering and Four Wave Mixing in Wavelength-Division Multiplexed Systems. Optics Communications, 250, 191-201. https://doi.org/10.1016/j.optcom.2005.02.011

[17] Sanchez-Lara, R., Alvarez-Chavez, J.A., et al. (2015) Threshold and Maximum Power Evolution of Stimulated Brillouin Scattering and Rayleigh Backscattering in a SM Fiber-Segment. Laser Physics, 25, Article ID: 035103. https://doi.org/10.1088/1054-660X/25/3/035103

[18] Hekmat, M.J., et al. (2013) Study of the Stimulated Brillouin Scattering Power Threshold in High Power Double-Clad Fiber Lasers. Laser Physics, 23, Article ID: 025104. https://doi.org/10.1088/1054-660X/23/2/025104

[19] Kovalev, V.I. (2014) Peculiarities of the Single and Repetitive Pulse Threshold Pump Power for Stimulated Brillouin Scattering in Long Optical Fibres. Laser Physics, 24, Article ID: 085407. https://doi.org/10.1088/1054-660X/24/8/085407

[20] Tithi, F.H., et al. (2012) Compensation of SBS Using Cross-Phase Modulation in WDM Transmission System. 7 th International Conference on Electrical \& Computer Engineering, Dhaka, 39-42. https://doi.org/10.1109/ICECE.2012.6471479

[21] Sanchez-Lara, R., Alvarez-Chavez, J.A., et al. (2014) Cascaded Raman Generation of the N'th Stokes in Single Mode-Fibers. Optical Engineering, 53, Article ID: 075102. 